\title{
On vaccination programs in the $E U$ Member States: the case of the human papilloma virus
}

\author{
Reyes Lorente ${ }^{1} \cdot$ Fernando Antonanzas ${ }^{1}$
}

Published online: 7 December 2016

(c) Springer-Verlag Berlin Heidelberg 2016

Vaccines are a specific kind of drug that help modify the immune response to an external agent, usually a bacteria or a virus, but also to any other entity such as pollen. Public health is mostly concerned with the effects of transmissible diseases, and hence vaccines are a key tool for coping with these conditions, making contagion-transmission difficult and even helping eradicate some of the agents, as was the case with smallpox. In this sense, in December 2014, The Official Journal of the EU published the European Council conclusions "On vaccination as an effective tool in public health". This text acknowledged that the EU would back national policies that target the most severe and common diseases to guarantee "a high level of protection of human health". The conclusions also stated that MS are the incumbent bodies that decide on the vaccination policies, remarking the existence of different aid programs within the EU such as those targeting some vulnerable groups across the regions and the mobility of the citizens. The same Council invited its Member States (MS) "to continue improving their national vaccination programs and to strengthen national capacity to carry out evidence-based cost-effective vaccination, including the introduction of new vaccines when considered appropriate" [1].

Furthermore, the European Council is empowered to establish recommendations to promote the implementation of health policies, to improve vaccination levels, to actively increase the education of the population in favor of vaccination, to train health care professionals, and to elaborate reports on the epidemiological situation as well as on the degree of implementation of the vaccination programs and

\footnotetext{
Fernando Antonanzas

fernando.antonanzas@unirioja.es

1 University of La Rioja, Logrono, Spain
}

their coverage levels. These activities are already currently developing, and will do so in the future, with the collaboration of the European Center for Disease Prevention and Control. However, the funding and management of vaccination programs correspond to MS; in other words, the European Council recommends, but the final decisions need to be adopted at the country level or even at the regional level, as is the case in some decentralized countries, such as Spain.

The implementation of health policies is related to the diagnosis of a specific situation, which is framed in a defined period and geographical context. Nevertheless, some health interventions, such as prophylactic vaccines, have short- as well as long-term effects, which generate uncertainty on future health outcomes with regard to not producing the anticipated results. The long vaccination tradition among MS together with recent EU recommendations have meant that, currently, all MS use vaccination to prevent severe and lethal conditions. However, according to the information collected by the European Centre for Disease Prevention and Control, vaccination calendars, target populations, and coverage levels are rather heterogeneous among MS, although for child vaccination programs, a higher homogeneity has been found [2]. In this sense, to improve the situation regarding child vaccination, the European Council had also published a set of recommendations (see 2011/C 202/02) inviting MS to share their experiences and best practices to increase the coverage of the programs.

The European Council requested that the European Commission continued the information exchange as well as the activities favoring the improvement of public health. The European Commission channels through the General Direction of Health and Food Safety the management of the promotion of public health. This institution helps MS 
maintain and improve vaccination rates by facilitating the introduction of vaccines within the EU and promoting their administration to each target group.

It is remarkable that the EU capacity to influence national policies to prevent transmissible diseases is still limited due to the sovereignty of MS in this area. Interestingly, there exists a reinforced influence of institutions encouraging more actions to prevent infection, such as the Regional Office for Europe of the World Health Organization [3] through its European Vaccine Action Plan 2015-2020, that is also promoting different policies with MS to procure "a European region free of vaccine-preventable diseases, where all countries provide equitable access to high-quality, safe, affordable vaccines and immunization services throughout the life course".

A rather odd phenomenon has been recently observed in the European population. In opposition to this EU policy favoring vaccination programs, there exists a growing opinion among citizens deeming vaccines as unnecessary, as public health levels achieved in many EU countries are high-but we must acknowledge that these high levels have been reached in part by vaccination programs too. This perception, together with the inherent side effects of these preventative measures, also contributes to reduced vaccination coverage. This is becoming a problem, and it is probably one of the reasons why some transmissible diseases have a growing incidence, higher than in the recent past. Ironically, society as a whole becomes more sensitive to transmissible diseases, and consequently to vaccines, when the risk of a pandemic seems to challenge public health, when some latent conditions are reactivated or when the appearance of new diseases can affect MS welfare (as noticed in the cases of ebola, zika, avian influenza, etc.). Therefore, European institutions need to insist on the necessity of immunization to counterbalance these behaviors by highlighting the risks derived from transmissible infections, and MS have to provide the means to achieve the desired prophylactic results.

\section{The case of the human papilloma virus (HPV) vaccination program}

Some vaccines are more socially acceptable than others. However, other vaccines trigger a social and health discussion about their convenience, even reaching political grounds. When some epidemiological and clinical elements of the conditions caused by the infections as well as the characteristics of the vaccine itself are not either severe or clear, then MS tend to differ in the way they adopt the decisions related to the implementation of vaccination programs. An example can be found in HPV. This vaccine was initially approved by the American Food and Drug
Administration in 2006 (both the bivalent and tetravalent prophylactic versions) and it was rapidly authorized by many countries. Since its approval it has been the object of many scientific studies (see, for instance, the review by $\mathrm{Lu}$ et al. [4]), together with an aggressive advertising campaign strategy unusual for this type of drug; this vaccine has also been supported by many medical societies across the world, although some will not recommend its use until there is more precise effectiveness information. The main objective of the communication efforts by pharmaceutical companies was to make the population sensitive to the necessity of its administration to avoid cervical cancer (although the HPV vaccine can also have an influence in other organs and in other conditions besides cancer development).

This vaccine has also been analyzed in the context of its adverse events, which seem to be already well known through the EU pharmacovigilance network (so far, less than one in 10,000 vaccinated persons develop bronchoconstriction with severe shortness of breath; it has also been observed that the adverse events tend to have a lower incidence in persons aged between 24-45).

The prophylaxis VPH HPV vaccine is currently administered in more than 40 countries in the world, the majority of them belonging to the higher income groupwith the exception of few countries, such as Ruanda. Among the MS, as in other jurisdictions, there is a vast acceptance of promoting the vaccination HPV program for women at early ages to increase the effectiveness (direct immunity) of the vaccine; that is the reason why the most frequent practice is to vaccinate girls aged 11-14 years, although some countries, such as Belgium, Denmark, the Netherlands and the United Kingdom offer a catch-up until 18 years too, or even beyond this age, as is the case in Austria, Czech Republic, France, Greece and Liechtenstein, for instance $[5,6]$.

In the EU, Austria was the first MS that implemented this vaccination policy, targeting both men and women, but lacking a public funding strategy [4]; this country also was the first to recommend the nonavalent vaccine of HPV (the newest version was authorized in the EU in 2015). Currently, the issues under discussion refer to the selection of older target groups, together with the possibility of extending the program to men too (although the vaccine is also authorized for men, few countries in the world have adopted this policy), and to the administration of the newest nonavalent version versus the former bivalent and tetravalent ones. Probably in the near future, when the therapeutic vaccine becomes authorized, a new decision regarding the global strategy about this infection will have to be adopted.

It is obvious that vaccination programs have an economic dimension, and MS have followed several approaches (systematic, opportunistic, fully or partially reimbursed) to control budgets. This dimension becomes 
more relevant in the case of the HPV vaccine as it has a unitary cost higher than the majority of existing vaccines. Because of this, for example, Belgium and France opted for partial reimbursement and opportunistic programs [5] while others preferred total public funding and universal coverage with systematic programs, as was the case in the Spanish regions; however, the selection of the vaccination programs and calendars is an evolving issue and the decisions adopted by MS have changed over time, and likely will do so in the future. It is also interesting to remember that HPV vaccination programs have been analyzed under the efficiency criterion, with the help of economic evaluation studies, yielding costs per quality adjusted life year (QALY) below 30,000 euros in the majority of the cases (as is well known, this ratio is a measure of the efficiency but does not guarantee the affordability of universal programs). This cost per QALY ratio is subject to the design of the simulation models used to investigate the efficiency, as well as the data used to populate these models, and significant variations are found depending on these inputs.

Given the complexity of HPV infection and its consequences on health, some elements related to this vaccine (some of them are also relevant for other immunization programs) probably need new specific assessments to better support health decisions. Among them, we list the following:

(i) Knowledge about the epidemiology of the infection and, derived from this, the actual preventive effect of the vaccine as a tool for reducing the incidence of cervical cancer, which usually takes between one and two decades after the infection to show symptoms (if the infected patient finally develops this cancer),

(ii) The duration of the total preventive effect of the vaccine and its immunity levels during the patient's life,

(iii) The dynamics of the infection across populations accounting for collective immunity too, and

(iv) The additional outcomes of other prophylactic measures related to this infection.

Data about these elements have to be collected, if already available-otherwise, they need to be directly investigated, and carefully analyzed-as a first step in guaranteeing the success of decisions about how to design HPV vaccination policies.

\section{Concluding remarks}

As we have illustrated through the case of HPV, the diversity of decisions related to the implementation of vaccination programs vary with differences in healthcare systems across MS and their strategies in adopting decisions, as well as with the characteristics of the incidence of the infection and the possibilities of implementing other preventative policies.

The variety of national health systems leads to differences in vaccination calendars for the vaccines already authorized in the EU, reimbursement mechanisms, vaccination approaches (systematic, opportunistic, universal, etc.), and so on, which will make difficult-if not impossible - to eradicate or at least significantly reduce some transmissible diseases that affect societies. Since viruses and bacteria do not know boundaries, the invitations of the European Council to both the European Commission and to the MS to facilitate access to vaccination programs as well as to coordinate the strategies for coping with the infections must resonate more. Reinforcing EU public health policy has already achieved positive health outcomes and by its nature is a necessary activity to keep moving things forward. It would be desirable to have a strategic framework on vaccination in the EU such that programs could be implemented without the immediate pressure of emergency challenges, assessing the pros and cons on scientific foundations - epidemiological, clinical and economic, and generating decisions binding to all MS so that viruses and bacteria would have more difficulties infecting persons within this geographic area.

\section{References}

1. The Council of the European Union. Council conclusions on vaccinations as an effective tool in public health (2014/C 438/04). Off. J. Eur. Union (2014)

2. European Centre for Disease Prevention and Control (ECDC). Human papilloma virus. Scientific Report (2012). http://ecdc. europa.eu/en/Pages/home.aspx. Accessed 26 Oct 2016

3. Regional Office for Europe of the World Health Organization. European vaccine action plan (2015-2020). http://www.euro.who. int/_data/assets/pdf_file/0007/255679/WHO_EVAP_UK_v30_ WEBx.pdf?ua=1. Accessed 04 Nov 2016

4. Lu, B., Kumar, A., Castellsagué, X., Giuliano, A.R.: Efficacy and safety of prophylactic vaccines against cervical HPV infection and diseases among women: a systematic review and meta-analysis. BMC. Infect. Dis. 11, 13 (2011). doi:10.1186/1471-2334-11-13

5. Bosch, F.X., Broker, T.R., Forman, D., Moscicki, A.B., Gillison, M.L., Doorbar, J., et al.: Comprenhensive control of human papillomavirus infections and related diseases. Vaccine 31(S7), H1-H31 (2013). doi:10.1016/j.vaccine.2013.10.003

6. Arbyn, M., de Sanjosé, S., Saraiya, M., Sideri, M., Palefsky, J., Lacey, C., et al.: EUROGIN 2011 roadmap on prevention and treatment of HPV-related disease. Int. J. Cancer 131, 1969-1982 (2012). doi:10.1002/ijc.27650 